\title{
An Application of Machine Learning in the Domain of Loan Analysis ${ }^{1}$
}

\author{
José Ferreira* \\ Joaquim Correia* \\ Thomas Jamet** \\ Ernesto Costa* \\ joseferreira@uc.pt \\ quim@lia.uc.pt \\ thomas@isoft.fr \\ ernesto@uc.pt \\ * Lab. de Informática e Sistemas, Universidade de Coimbra, 3000 COIMBRA, PORTUGAL \\ **Isoft, Chemin du Moulon, 91400 ORSAY, FRANCE
}

\begin{abstract}
Making decisions on whether to give or not a financial support to an industrial project is a very common and yet very complex task. Financial institutions need much expertise to deal with the large amount of information that has to be considered for this process. An expert system based approach seenis to be an interesting solution to the problems raised by this type of application. Knowledge acquisition is, however, a very time consuming task. We have used APT, a multistrategy learning system, as a knowledge elicitation tool in the domain of loan decision. We describe the process of building and refining a knowledge base and compare the results of our approach to a conventional expert system.
\end{abstract}

Keywords: generalization, knowledge acquisition, machine learning applications

\section{Introduction}

The analysis of large scale projects that ask for financial support is a regular activity in banking institutions. A loan process comprises five steps: proposal, proposal analysis, decision, contract and amortization.

The most important step is the decision one, since the others are mostly administrative steps. The decision process begins with an analysis of the documentation provided by the project's promoter, involving an economist and an engineer that are responsible for the first appreciation of the loan request. Then, experts will visit the plant, if the enterprise exists. Finally, the decision is taken. All these activities involve high costs and require a lot of expertise. To deal with these needs in expertise, an expert system, SPAC [5], has been developed at Bank Caixa Geral de Depósitos, in collaboration with the University of Lisbon.

As usual in the process of building knowledge based systems, acquiring the knowledge for SPAC was the toughest and longest task. To overcome these difficulties, we have used APT [4] as an automatic knowledge base builder. We wanted to test APT's applicability in this domain and verify if there could be significant gains in time and in the dimension of the knowledge base.

To implement this application, we have had the collaboration of the experts from the Bank that provided SPAC and gave some information regarding the domain in hand. They also have validated the results obtained after we have used APT.

In this paper we present the results of our experiment. We begin with brief descriptions of both SPAC and APT. Next, we describe the domain. We then present

${ }^{1}$ This work was partially supported by CEC, through ESPRIT-2 contract 2154 - MLT 
APT at work and conclude with a summary of the results and some comments on the validity of the approach.

\section{The SPAC Expert System}

SPAC is a standard forward chaining rule based system. It took about two years to develop to the current state, it currently contains about 450 rules and is now being tested at the bank.

Several sources were used for knowledge elicitation:

- readings on loan guide books and loan processes;

- structured and unstructured interviews;

- responses given by experts to elicitation grids for uncertainty coefficients.

Rules in SPAC are of the form:

MakeRule(decision20, ((enterprise:value \#= existing) AND

(promoter_profile:value \#= weak) AND

(project_quality:value \#= satisfactory) AND

(econ_financ_feasibility:value \#= weak) AND

(guarantees:value \#= good),

$\{$ decision:value $=$ no; decision: certainty $=1.0 ;\}$ );

The meaning of the rule above is: when promoter's profile is weak, project's quality is satisfactory, economic and financial feasibility is weak, guarantees of payment are good and the enterprise exists, the decision to financially support the project is no with a certainty value of 1.0 .

\section{APT}

APT is a multistrategy learning system that is able to learn general problem solving rules from one single example given by one expert, provided it has some initial knowledge of the domain. For a complete description of the system see [4].

APT uses a domain theory, in the form of hierarchies of concepts, some of them having relations associated to other concepts in the theory and properties associated to values (numerical ones, for example). We show in figure 1 a partial representation of some of the concepts that are considered in the session we present next. Due to the extension, we do not present the complete hierarchy.

The expert initially proposes a problem. If there are any rules applicable to the problem, they are presented. If there are no rules, or the user rejects the proposed ones, he is asked for a particular solution for the problem. The solution is normally a decomposition of the problem into simpler ones. We call this a reduction. Once this is given, APT looks for relations among the concepts appearing in the problem and in the reduction. These are proposed as explanations, in the sense of causal justifications of why the reduction is suitable for the problem. The explanations accepted by the expert provide a lower bound for a rule that APT will try to generalize. It will then propose a set of examples, following a smallest generalization step strategy [2], allowing to generalize one concept at a time and verifying that any further generalization is justified by the preceding steps. At the end, one or more rules will be learned. The expert accepts, rejects or adjusts them. If adjustment has to take place, APT enters a phase that allows for guided refinement of the domain theory [4]. As a 
result, one or more rules will be learned and the domain theory will incrementally be refined and completed.

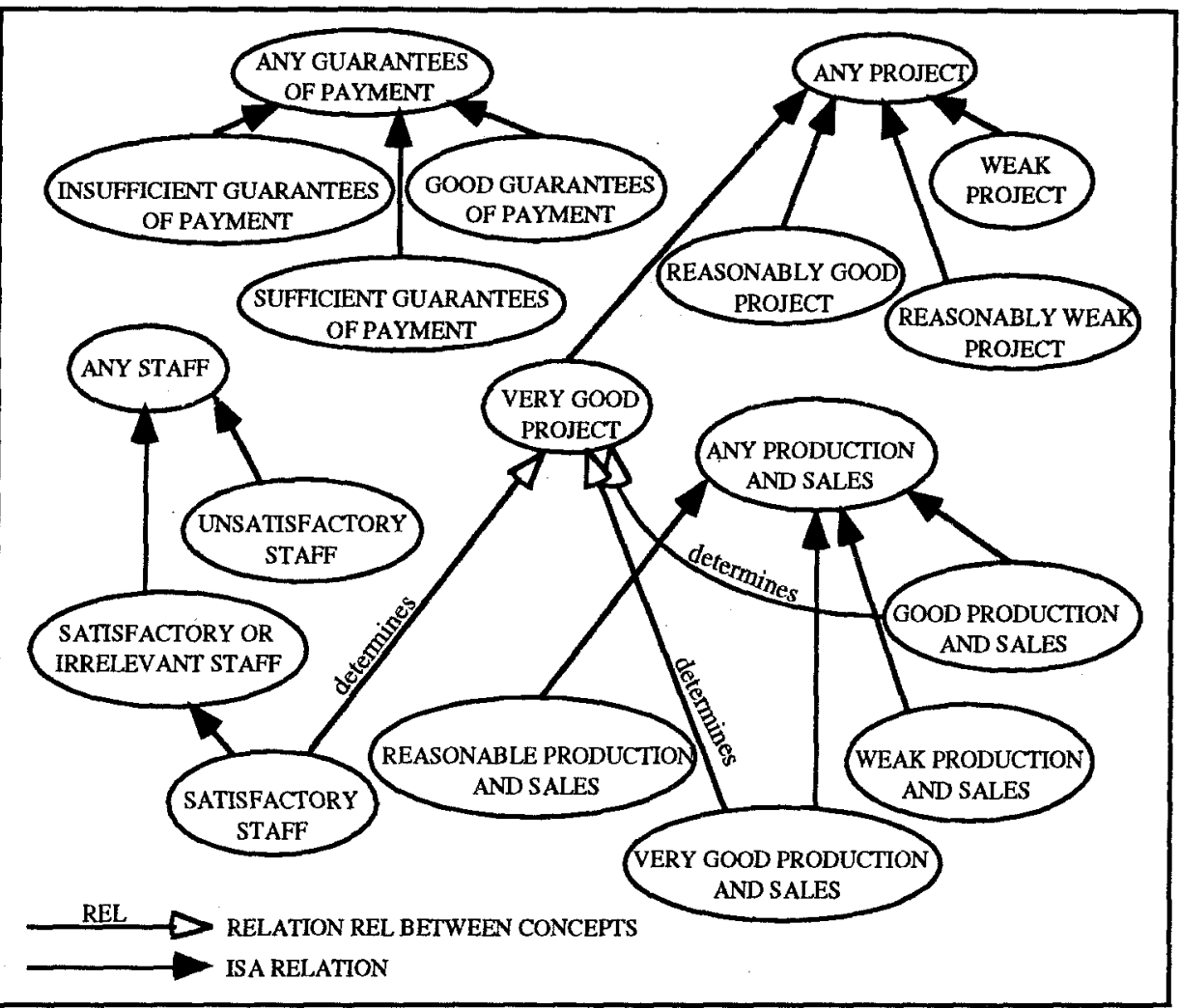

Fig. 1. Part of the initial domain theory.

\section{Loan Analysis And Decision}

In figure 2, we present a partial taxonomy of the problems in the domain. Square boxes are filled with non-elementary problems, whereas elliptical delimiters with elementary problems. As shown in the figure, several sub-problems are not elementary, but due to its extension we do not present the complete hierarchy of problems.

Making decisions on loan support involves several uncertainties, even for the experts in the domain. Deciding on the leadership capacity of a project's promoter is rather fuzzy and necessarily introduces uncertainty in the results. SPAC accounts for this fuzziness by using certainty factors associated to each rule. APT however, can not use this type of information. The rules acquired by APT will, therefore, disregard this incertitude. Nevertheless, APT's results are quite satisfactory. We justify this statement by noticing that in SPAC, certainty factors are propagated over the rules involved in a decision process and assign a confidence degree to the result. They are never used to inhibit or emphasize the applicability of a rule, which means that every applicable rule will be considered, regardless its results. So, for both SPAC and APT, the final decision is the same, differing only in the confidence degree. 


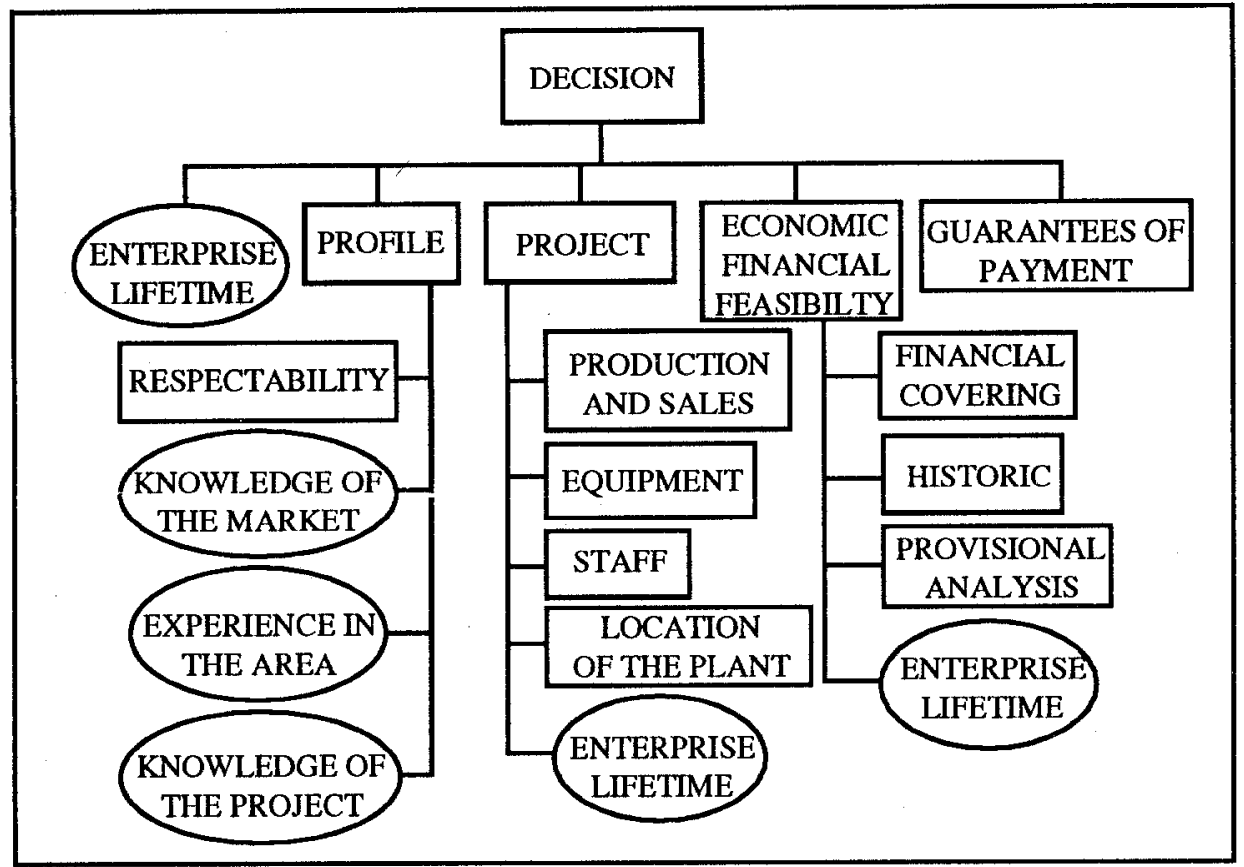

Fig. 2. Partial taxonomy of problems in the domain of loan decision.

\section{APT At Work}

We can not show the session with APT, because it is very long. See [1] for a detailed description. We will summarize some aspects of the interaction with APT.

Consider the problem of evaluating the quality of a project, in the case of a new enterprise with a very good production and sales policy, considering the plant has a good location and suitable equipment and staff is satisfactory.

As there are no rules for solving the proposed problem, the user is asked for a solution. He said that his particular project can be classified as very good.

APT now tries to establish causal relations between the concepts in the problem and those in the solution. It finds the relation determines between the considered concepts and builds explanations for the problem/solution pair around this relation. All proposed explanations were accepted in this session. Starting with the problem, the solution and the explanations, a rule is created. The explanations define the applicability condition for the rule, which can thus only be applied to the particular problem. The generalization process now begins. It is considered that the problem given by the user is an instance of a more general class of problems. It is this class of problems that we are trying to learn how to solve. APT builds a generalization lattice, that defines the generalization possibilities for each concept in the rule. It will then walk through the lattice, one step at a time, generating and proposing more general examples. For a complete description of the generalization process see [2]. The user classifies the proposed examples as valid or invalid instances of the class of examples he wants APT to learn. For this particular problem, 18 examples were proposed, 15 of them accepted and the other 3 rejected. 
At the end of the generalization phase, an over general rule was acquired, because one concept was over generalized. Some of its sub concepts produce positive instances of the class of problems, others don't. The domain theory has to be refined by grouping the "positive concepts" as sub concepts of a new concept and the "negative concepts" as sub concepts of a different new one.

The learned rule said: when the enterprise is new or existing, the production and sales policy is very good or good, the location of the plant is good, with suitable or irrelevant equipment, satisfactory or irrelevant staff, the project is very good.

\section{Evaluation}

Our primary concern when using APT as a knowledge acquisition tool in this particular domain was to observe the results in terms of how many rules we would have and how much of the application cculd be modeled. We had strong-expectations on the comparison between the number of rules we would have at the end and those in SPAC, because these are very particular. We also wanted to test APT's applicability to this kind of domains, specially involving decision tasks.

\begin{tabular}{|c|c|c|}
\hline PROBLEM & $\begin{array}{c}\text { \# SPAC } \\
\text { RULES }\end{array}$ & $\begin{array}{c}\text { \# APT } \\
\text { RULES }\end{array}$ \\
\hline DECISION & 44 & 5 \\
\hline PROFILE OF A NEW PROMOTER & 17 & 10 \\
\hline PROFILE OF EXISTING PROMOTER & 18 & 6 \\
\hline PROJECT EVALUATION & 48 & 11 \\
\hline ECONOMIC AND FINANCIAL FEASIBILITY & 34 & 6 \\
\hline GUARANTEES OF PAYMENT & 15 & $\left({ }^{*}\right)$ \\
\hline RESPECTABILTYY OF NEW PROMOTER & 3 & 2 \\
\hline RESPECTABILITY OF EXISTING PROMOTER & 4 & 3 \\
\hline MANAGEMENT CAPACITY & 26 & 9 \\
\hline PRODUCTION AND SALES & 38 & 14 \\
\hline LOCATION OF THE PLANT & 36 & 9 \\
\hline EQUIPMENT & 37 & 9 \\
\hline STAFF & 15 & 6 \\
\hline COMMERCIALIZATION & 40 & 4 \\
\hline RAW MATERIAL & 22 & 5 \\
\hline PRODUCT-MARKET RELATION & 58 & 26 \\
\hline
\end{tabular}

(*) It was not possible to execute the sessions regarding this particular sub-problem, because the evaluation of the guarantees for payment was not provided by the bank. Formulas for this evaluation are secret.

APT has proved its capabilities as a knowledge elicitation tool in the domain. Because it allows for incremental refinement of the domain theory, it avoids the necessity of a complete formulation of a domain theory right from the beginning. It is normally very difficult to produce such complete, error-free formulation. The strategy used by APT, generalizing one concept at a time is a very friendly one. It prevents for extraneous generalizations due to the incapacity of classifying very general examples. We have noticed that the number of rules learned by APT is much lesser than those in SPAC, as shown in the table. More important than the number is the quality of the rules. Experts from the bank have validated the resulting rules. They 
agreed on their quality and, moreover, they realized that SPAC would profit if the domain could be structured as APT's domain theory was. This is now being done.

We have also noticed that the deeper the hierarchies of concepts involved in one problem are, the greater the difference between the number of rules of APT and the number of rules in SPAC. This is because we can generalize further in the hierarchies, so each APT rule covers more SPAC rules.

Comparison of the time spent to build the application could not be considered, because we already had a knowledge base. But, since we began with no knowledge about the application domain, we have had some of the problems that knowledge engineers normally have to face when eliciting knowledge. We "interviewed" the SPAC rules to build the domain theory and we used SPAC itself to classify the examples proposed by APT.

\section{Conclusions}

We found that the use of learning systems as APT for knowledge elicitation can be a very interesting way to overcome the problem of extracting knowledge from experts. The improvements achieved with such learning systems can be of several types:

- the initial domain theory does not need to be complete, since the learning sessions will be responsible for the detection of missing knowledge, and will automatically correct it, with the guidance of the user;

- the expert (or the knowledge engineer) does not have to think of all cases at once, only a few examples of the classes of cases he can have in the domain, since the system will generate the others, by looking for analogous examples; understand.

- the interactive characteristic of the system makes it friendly and easy to

\section{References}

1. Coimbra, Isoft, and LRI: Case Study on Loan Analysis. Technical Report, MLT ESPRIT Project P2154, November 1992.

2. NEDELLEC C.: A Smallest Generalization Step Strategy. In: Proceedings of the Eighth International Workshop on Machine Learning, pp 529-533, June 1991.

3. NEDELLEC C.: How to Specialize by Theory Refinement. In: Proceedings of Tenth European Conference on Artificial Intelligence, August 1992.

4. Isoft, LRI, and Coimbra: User's guide to APT. Deliverable 4.2, MLT ESPRIT Project P2154, September 1991.

5. P. J. XARDONÉ and E. P. LEITÃO: SPAC - Sistema Pericial para Análise de Crédito. Faculdade de Ciências, Universidade de Lisboa, 1989. 